\title{
PERCEPÇÕES DE GESTANTES RIBEIRINHAS SOBRE A ASSISTÊNCIA PRÉ-NATAL
}

\author{
Alexandre Aguiar Pereira ${ }^{1}$, Fabiane Oliveira da Silva ${ }^{2}$, Gisele de Brito Brasil ${ }^{3}$, Ivaneide Leal Ataíde Rodrigues ${ }^{4}$, \\ Laura Maria Vidal Nogueira ${ }^{4}$
}

RESUMO: Objetivos: o estudo objetivou identificar a importância do pré-natal para gestantes ribeirinhas e conhecer suas expectativas, discutindo esses aspectos com vistas a melhorar a qualidade da assistência de saúde a essas mulheres. Método: estudo qualitativo descritivo, cujos dados foram coletados de agosto a outubro de 2016, por entrevistas individuais com 15 gestantes que realizavam pré-natal na Unidade de Saúde da Família da Ilha do Combú, localizada ao Sul da cidade de Belém, Pará, e analisados por meio da técnica de análise temática. Resultados: dos depoimentos analisados, originaram-se duas categorias: A importância do pré-natal como garantia de saúde para a mãe e para o bebê, e O pré-natal como fortaleza e fonte de aprendizado. Conclusão: conhecer o significado do pré-natal na vida das gestantes, considerando suas particularidades, oferece subsídios para que o profissional de saúde, especialmente o enfermeiro, possa ajudar em possíveis lacunas, garantindo assistência pré-natal de qualidade.

DESCRITORES: Assistência pré-natal; Gestante; Percepção; Enfermagem; Saúde da Mulher.

\section{PERCEPTIONS OF RIVERINE PREGNANT WOMEN ON PRENATAL CARE}

\begin{abstract}
Objectives:The present study aimed to identify the importance of prenatal care for riverine pregnant women andunderstand their expectations, by discussing these aspects in order to improve the quality of the healthcare provided to this population. Method: Qualitative descriptive study that used data collected from August to October 2016, through individual interviews with 15 pregnant women who received prenatal care at the Family Health Unit of Ilha do Combú, in the south of the city of Belém, state of Pará, and analyzed with the use of thematic analysisResults:Two categories emerged from the statements of the participants. The importance of prenatal care to protect the healthofmothers and babies, and Prenatal care as a strengthand source oflearning. Conclusion:The study aimed to gain insight on the meaning of prenatal care in the lives of pregnant women, considering their particularities, make such knowledge available to health professionals, especially nurses, so that they can contribute to solve possible gaps and ensurehigh quality prenatal care.
\end{abstract}

DESCRIPTORS: Prenatal care; Pregnant woman; Perception; Nursing; Women's Health.

\section{PERCEPCIONES DE GESTANTES RIBEREÑAS ACERCA DE LA ASISTENCIA PRENATAL}

RESUMEN: Objetivos: el estudio tuvo el objetivo de identificar la importancia del prenatal para gestantes ribereñas y conocer sus expectativas, discutiendo esos aspectos para mejorar la calidad de la asistencia de salud a esas mujeres. Método: estudio cualitativo descriptivo, cuyos datos se obtuvieron de agosto a octubre de 2016, por medio de entrevistas individuales con 15 gestantes que realizaban prenatal en la Unidad de Salud de la Familia de la Isla del Combú, ubicada al sur de la ciudad de Belém, Pará. Se los evaluó por medio de la técnica de análisis temático. Resultados: de los testimonios analizados, se originaron dos categorías: La importancia del prenatal como garantía de salud para la madre y para el bebé, y El prenatal como fuente de fuerza y de aprendizaje. Conclusión: conocer el significado del prenatal en la vida de las gestantes, considerando las particularidades, da subsidios para que el profesional de salud, especialmente el enfermero, pueda ayudar en posibles lagunas, garantizando asistencia prenatal de calidad.

DESCRIPTORES: Asistencia prenatal; Gestante; Percepción; Enfermería; Salud de la Mujer.

${ }^{1}$ Enfermeiro. Residente de Enfermagem em Atenção à Saúde da Mulher e da Criança. Universidade do Estado do Pará. Belém, PA, Brasil.

${ }^{2}$ Enfermeira. Mestranda em Enfermagem. Universidade do Estado do Pará/Universidade Federal do Amazonas. Belém, PA, Brasil.

${ }^{3}$ Enfermeira. Mestre em Enfermagem. Universidade do Estado do Pará/Universidade Federal do Amazonas. Belém, PA, Brasil. ${ }^{4}$ Enfermeira. Doutora em Enfermagem. Docente de Enfermagem. Escola de Enfermagem Magalhães Barata. Belém, PA, Brasil. 


\section{INTRODUÇÃO}

O pré-natal pode ser entendido como assistência de saúde prestada à mulher gestante, a fim de garantir gestação adequada e segura, por meio do acompanhamento de saúde de qualidade. O Caderno de Atenção ao Pré-natal de Baixo Risco ${ }^{(1)}$ afirma claramente que o objetivo do acompanhamento prénatal é garantir o desenvolvimento da gestação, sem impacto para a saúde materna, permitir o parto de recém-nascido saudável, além de abordar aspectos psicossociais e realizar atividades educativas e preventivas.

Para assegurar um pré-natal de qualidade, preconiza-se que a gestante receba, no mínimo, seis consultas, com acompanhamento intercalado entre médico e enfermeiro. A atuação do enfermeiro na realização do pré-natal tem sido fundamental para a melhoria da assistência às gestantes, pois favorece o aumento da cobertura pré-natal e tem contribuído significativamente para a humanização da assistência prestada ${ }^{(2)}$.

O acompanhamento da mulher no ciclo grávido-puerperal deve ser iniciado o mais precocemente possível, e só se encerra com o seu retorno e do recém-nascido ao serviço de saúde entre 7 a 10 dias de puerpério e uma consulta médica ou de enfermagem com 42 dias pós-parto ${ }^{(1)}$. Partindo deste princípio, é direito de toda mulher receber o acompanhamento de pré-natal durante a gravidez, independente de sua origem, cultura ou sociedade pertencente, sejam elas do meio urbano ou rural ${ }^{(3)}$.

Nas últimas duas décadas têm-se proposto indicadores de adequação para avaliar a qualidade dos cuidados pré-natais, incorporando outros elementos além da época de início do acompanhamento e do número de consultas recebidas ${ }^{(4)}$. De maneira geral houve melhorias na atenção à saúde das gestantes, contudo, considerando a diversidade que caracteriza o Brasil, seja em relação às condições socioeconômicas e culturais, seja em relação ao acesso às ações e serviços de saúde, entende-se que o perfil epidemiológico da população feminina apresente diferenças importantes de uma região a outra do país ${ }^{(5)}$.

Tradicionalmente, as comunidades ribeirinhas são compostas de vários agrupamentos familiares, em casas de madeiras, adaptadas ao sistema de cheias e vazantes dos rios, dispersas ao longo de um percurso fluvial. Vivem, em sua maioria, à beira dos rios, igarapés, igapós e lagos, estando isolados, com pouco ou restrito acesso à mídia escrita e falada ${ }^{(6)}$. É nesse contexto que se encontra a mulher ribeirinha e o atendimento pré-natal, que muitas vezes é dificultado pelas condições socioeconômicas dessas mulheres.

Em virtude do isolamento de seus núcleos populacionais, resultado da distribuição dispersa, a realidade dessas populações revela grande desigualdade de acesso aos serviços públicos de saúde em comparação com as áreas urbanas. Assim, para a promoção de atenção acessível, humanizada, e que seja capaz de tornar a busca pelo cuidado em prática frequente, é necessário compreender as formas de ligação entre as mulheres residentes em áreas não urbanas e os serviços de saúde ${ }^{(7)}$.

Dessa forma, tendo em vista a importância do pré-natal e buscando entender a visão que as gestantes ribeirinhas têm sobre ele, definiu-se como questão de pesquisa deste estudo: Quais as percepções de gestantes ribeirinhas a respeito do pré-natal? Para responder a essa questão estabeleceu-se como objetivos: Identificar a importância do pré-natal para gestantes ribeirinhas; Conhecer as expectativas dessas gestantes sobre o pré-natal; e Discutir esses aspectos com vistas a melhorar a qualidade da assistência de enfermagem a essas mulheres.

\section{MÉTODO}

Trata-se de um estudo descritivo, de natureza qualitativa, que teve como cenário a Unidade de Saúde da Família (USF) da Ilha do Combú. Essa ilha possui uma área de aproximadamente 32 km², e está localizada ao Sul da cidade de Belém, no estado do Pará, a aproximadamente 1,5 km de distância da costa. O local se divide em seis microáreas, com cerca de 530 famílias cadastradas no serviço de saúde. Algumas dessas microáreas possuem escasso acesso, ficando isoladas devido ao ciclo das marés. A USF conta com uma equipe de saúde completa, composta de cinco Agentes Comunitários de Saúde 
(ACS), uma enfermeira, um médico e três técnicos de enfermagem.

Os dados foram produzidos no período de agosto a outubro de 2016, por meio de entrevista individual com 15 gestantes, dentre 21 que realizavam o acompanhamento pré-natal na USF do Combú. As entrevistas foram encerradas ao chegar-se à saturação dos dados, critério que permite estabelecer a validade de um conjunto de dados quando nenhum novo elemento é encontrado e o acréscimo de novas informações deixa de ser necessário, pois não altera a compreensão do fenômeno estudado ${ }^{(8)}$. $\mathrm{O}$ formulário aplicado estava dividido em duas partes: a primeira, com o perfil das gestantes e a segunda, com cinco perguntas abertas que exploraram o objeto de estudo.

Como critério de inclusão foi considerado: grávidas de qualquer faixa etária, de qualquer microárea, cadastradas na USF do Combú, independente da idade gestacional, que realizassem acompanhamento pré-natal e que apresentassem condições físicas e mentais para responder ao instrumento para coleta de dados.

Inicialmente, o projeto foi apresentado à equipe da USF do Combú, com intuito de explicar o que seria feito e facilitar a abordagem com as gestantes. Estas foram convidadas de acordo com seu comparecimento às consultas e as entrevistas foram realizadas na USF ou no domicílio da gestante, conforme sua escolha, e sendo gravadas mediante o consentimento. A análise deu-se por meio da técnica de análise de conteúdo ${ }^{(9)}$.

Durante o desenvolvimento da pesquisa, foram considerados os preceitos éticos da Resolução $n^{\circ} 466 / 12$ do Conselho Nacional de Saúde ${ }^{(10)}$. Recebeu autorização Institucional e foi aprovada pelo Comitê de Ética em Pesquisa da Universidade do Estado do Pará, sob parecer no 1.574.224.

Às participantes foi apresentado o Termo de Consentimento Livre e Esclarecido, para que pudessem manifestar seu interesse de participar. $\mathrm{O}$ anonimato das participantes foi assegurado por meio do uso de código alfanumérico para identificação das entrevistas, com a letra $\mathrm{G}$ e o número sequencial das mesmas (G1, G2,...G15).

\section{- RESULTADOS}

\section{Perfil das Participantes}

A idade das entrevistadas variou entre 17 e 32 anos, predominando a faixa etária de 18 a 24, com nove participantes, sendo duas menores de 18 anos. Em relação à microárea de residência, sete residiam na microárea um, que é a mais próxima à USF conforme a divisão estratégica da ilha para o acompanhamento da população. Na escolaridade, predominou o ensino fundamental completo, com cinco participantes. A renda familiar predominante foi de um salário mínimo.

Em relação à idade gestacional, nove estavam entre sete e nove meses de gravidez. Houve oito primíparas. No que diz respeito ao acompanhamento de pré-natal, das sete gestantes que tinham mais de um filho, cinco delas iniciaram seu acompanhamento no primeiro trimestre de gravidez, inclusive da gestação atual. Todas as entrevistadas realizaram o pré-natal em suas gestações anteriores. A importância do pré-natal iniciado no primeiro trimestre de gravidez justifica-se pelo fato de serem mais frequentes nesse período a ocorrência das principais intercorrências obstétricas, como hemorragias e abortos, além da necessidade de realização de exames e avaliações para detecção de anormalidades precoces que possam interferir na saúde materna e fetal.

Para a análise dos dados foi aplicada a técnica de análise de conteúdo, obedecendo-se o passo a passo dessa análise. Iniciou-se pela transcrição das gravações, constituindo o corpus, do qual se procedeu a leitura flutuante, explorando o material para a identificação dos temas mais recorrentes em ocorrência e co-ocorrência. Os temas identificados foram agrupados de forma a atender aos objetivos do estudo, o que propiciou a organização de duas categorias temáticas: A importância do pré-natal como garantia de saúde para a mãe e para o bebê, e O pré-natal como fortaleza e fonte de aprendizado, que serão apresentadas e discutidas a seguir. 
Nesta categoria, evidenciaram-se as percepções das participantes a respeito da importância do pré-natal para a sua saúde e para a saúde do bebê. Ao serem questionadas sobre essa importância e o porquê, de maneira unânime, fizeram um conjunto de explicações que apontavam para a sua importância como estratégia para garantir e ofertar saúde.

Nas explicações, destacam-se expressões que atrelam as consultas de pré-natal ao acompanhamento da situação de saúde da mãe e do bebê e a necessidade desse acompanhamento para uma gestação saudável. A importância do pré-natal foi traduzida com expressões, como "se está bem", "minha saúde e a do bebê" ou similares por todas elas:

Sim, é muito importante! Para a saúde do bebê, para ver como está, saber se ele está bem.(G2)

É! É importante... é essencial, com certeza é importante sim! Porque assim eu vou cuidar não só da minha saúde como a da criança, entendeu? Aí eu posso evitar que tanto eu quanto meu filho venha ter outros problemas relacionados à saúde [...]. (G6)

O entendimento de que o pré-natal é de suma importância para combater doenças e complicações na gravidez também foi evidenciado. Para sete $(46,6 \%)$ delas, o pré-natal contribui para combater doenças que possam afetar sua gestação, assim como prevenir o aparecimento de complicações comuns da gravidez:

Eles passam exame e eu venho pra saber se vai dar alguma coisa, se vai aparecer alguma doença pra poder tratar. Por exemplo, no meu exame deu anemia e eu consegui tratar no sexto mês de gravidez. (G1)

Porque é através do pré-natal que eu consigo evitar todos os tipos de doença, infecção urinária, essas coisas, e saber o que eu tenho durante a gravidez e após a gravidez.(G9)

Foi destacada pelas gestantes a importância do acolhimento da equipe de saúde para a garantia de um atendimento com qualidade no pré-natal. Os depoimentos demonstraram o papel da equipe orientando, aconselhando e educando essas mulheres. Esse aspecto é citado em oito depoimentos:

[...] Coisas, assim, que eu nunca ia saber em casa e lá eles me ajudam, a nossa mãe dá alguns conselhos, mas outras coisas eu só aprendo lá [na USF]! As maneiras corretas, que muitas das vezes as mães ensinam, mas não é aquilo o correto, podemos dizer assim. Cada consulta que eu vou eles ensinam alguma coisa. (G6)

[...] É bom ter um acompanhamento médico, porque qualquer problema a gente vai, fala nossas dúvidas e eles explicam pra gente, como é, como não é, então eu acho muito importante sim. (G15)

Portanto, cabe à equipe de saúde, especialmente a de enfermagem, saber ouvir e acolher esse público, uma vez que o acompanhamento por meio das consultas objetiva também realizar a aproximação da gestante com o profissional, permitindo a criação de vínculo.

\section{O pré-natal como fortaleza e fonte de aprendizado}

Nesta categoria, discutem-se as expectativas manifestadas pelas participantes quanto ao pré-natal. Notou-se nos depoimentos que o pré-natal é visto como uma fortaleza e segurança para elas e seu bebê. Nessa ótica, manifestaram sua total confiança e esperança no programa de pré-natal para terem gestação e parto seguro. Para todas, o pré-natal assegura uma boa gestação e um bom parto no futuro:

Serve para prevenir a criança, para ter o parto bom, principalmente. [...] Porque sem ele a gente não se cuidaria muito bem. (G10)

Serve para acompanhar a saúde do bebê, para saber se está tudo bem comigo e com ele. É muito importante, porque ele me ajuda a saber como está meu bebê, se vai nascer bem, com saúde, essas coisas.(G14)

Uma expectativa bastante mencionada diz respeito à necessidade de outros profissionais para o acompanhamento pré-natal, além da realização de exames no próprio serviço, evitando-se, dessa forma, a busca desse atendimento na capital. Mencionou-se a necessidade de dentista, nutricionista, 
pediatra, ginecologista, e realização de exames clínicos e obstétricos na própria Unidade em oito depoimentos:

Acho que era bom ter pediatra, ter dentista, porque evitaria que a gente tivesse que ir pra Belém, e também ter outros especialistas para cuidar da nossa saúde. (G7)

O que eu achava melhor era vim pra cá mais médicos! Porque a gente precisa muito aqui... e exames também, porque evitaria da gente ir lá para Belém. [...] Isso melhoria muito a nossa situação. (G8)

Também se percebeu nas falas um atendimento satisfatório que elas buscavam a cada consulta. O pré-natal evidenciou-se como momento de esclarecimento de suas dúvidas e de sanar seus anseios. Nessa perspectiva, 11 afirmaram que esperavam ser bem atendidas e saírem satisfeitas das consultas:

Espero ter um atendimento satisfatório e me explicar a respeito de tudo sobre a gravidez. (G5)

Espero que me examine, que cuide do meu bebê, que me atendam bem. (G14)

Entende-se que as consultas de pré-natal tornam-se oportunas para a educação das gestantes, que almejavam receber orientações e conselhos. Nesse sentindo, observou-se em sete participantes esse anseio de ser aconselhada pelos profissionais e sair esclarecida dos atendimentos:

Eu espero ter um bom atendimento [...], eles tirem minhas dúvidas, me orientem e que me falem como está minha gravidez, se está tudo bem! Faz acompanhamento, aconselhamento e outras coisas. (G6)

Bom, o que eu espero que eles façam? Tirem minhas dúvidas é claro, com dificuldades que todas as grávidas têm. [...] Em muitas coisas, porque pra mim ele vai me orientar, me ensinar as coisas, porque eu tenho três filhos, mas tem muitas coisas que eu não sei, aí vão estar me orientado na hora da consulta. (G12)

\section{- DISCUSSÃO}

Os dados da pesquisa apontaram que as gestantes entrevistadas sentem a importância da assistência pré-natal para a gestação, expressam isso por meio de suas falas trazendo suas expectativas, anseios e necessidades quanto ao acompanhamento, acolhimento e cuidados de saúde da equipe da Unidade. Dessa forma, conhecer as relações que essas mulheres estabelecem com os serviços de saúde permanece como desafio necessário para promover o acesso à atenção humanizada que estimule a busca pelo cuidado de forma cotidiana ${ }^{(11)}$.

Entender a importância do pré-natal para as mulheres permite apreender o significado desse cuidado para elas, auxiliando no direcionamento da assistência prestada. É importante compreender o contexto cultural, a visão de mundo e as particularidades das pessoas, reconhecendo que, muitas vezes, elas as manifestam de formas peculiares, que precisam ser abarcadas na assistência, de modo a realizar o cuidado de forma mais efetiva ${ }^{(12)}$.

$\mathrm{Na}$ visão das participantes desse estudo, o pré-natal ajuda na detecção precoce de doenças e alterações gestacionais. E de fato, o acompanhamento pré-natal de qualidade configura ação voltada para esses aspectos, com o tratamento de intercorrências de saúde materna, colaborando para a redução de riscos tanto para a gestante quanto para o concepto ${ }^{(13)}$. Nesse sentido, esta atenção caracteriza-se desde a concepção até o início do trabalho de parto, de forma preventiva e tendo também como objetivos identificar, tratar ou controlar patologias, além de prevenir complicações na gestação e parto ${ }^{(14)}$. Percebeu-se, assim, essa compreensão e entendimento das mulheres a respeito do pré-natal.

Ressalta-se o papel da equipe para garantir a saúde das gestantes e do bebê, uma vez que ela é vista como a principal condutora desse processo. Uma das estratégias que pode facilitar a adesão ao acompanhamento pré-natal e reduzir o distanciamento entre profissional de saúde e gestante é a aplicação de novas metodologias de trabalho que humanizem a assistência e dêem um toque de originalidade e reconhecimento do outro no processo de cuidado ${ }^{(15)}$.

As equipes de saúde que atuam nas áreas ribeirinhas são mais reduzidas, decorrente das dificuldades de acesso, havendo um número limitado de profissionais de saúde, assim como de especialidades 
médicas, que permanecem concentrados nos grandes centros urbanos ${ }^{(6)}$. Esse entendimento esteve presente nos depoimentos das entrevistadas.

Os depoimentos também demonstraram que as participantes julgam a relevância do pré-natal de acordo com a atenção dada a elas. Esse aspecto também foi evidenciado em estudo que demonstrou que as entrevistadas relacionaram a importância do pré-natal à atenção dispensada a elas. Segundo os autores, o acolhimento à gestante é essencial no atendimento, pois inclui a recepção da mulher desde o primeiro contato com a unidade de saúde, responsabilizando-se por ela ${ }^{(16)}$.

O enfermeiro, mediante suas atividades, exerce papel importante às gestantes para que a adesão ao pré-natal seja contínua, posto que, como cuidador, ele se encarrega de oferecer ação integral para a saúde dessas mulheres e promover atividades que contribuam no favorecimento de uma gestação e nascimento saudáveis ${ }^{(17)}$.

Repensar a atenção pré-natal envolvendo os profissionais pressupõe novo olhar sobre o processo de trabalho em saúde e organização do serviço, por meio de protocolos que valorizem a competência técnico-científica de cada membro da equipe multiprofissional, oferecendo assim assistência de qualidade e humanizada à gestante ${ }^{(12)}$. A aquisição de novos conhecimentos científicos pode subsidiar o trabalho desses profissionais de ajudar a mulher a superar as dificuldades que essa condição impõe ${ }^{(18)}$.

Os resultados também evidenciaram o papel educacional do programa de pré-natal. A consulta pré-natal envolve procedimentos simples, podendo o profissional de saúde dedicar-se a escutar as demandas da mulher, transmitindo confiança para conduzir com autonomia a gestação e o parto. É necessário que o profissional esclareça as dúvidas geradas com muita clareza, de forma que a mulher se sinta segura ${ }^{(14)}$.

Nesse contexto, ressalta-se que a assistência pré-natal tem o objetivo de orientar e esclarecer sobre o parto e os cuidados com o recém-nascido. O pré-natal é um momento singular e oportuno para desenvolver ações educativas, podendo ser realizadas nas unidades de saúde, por intermédio de grupos de gestantes, salas de espera, ou individualmente. Essa estratégia de trabalho permite a integração de profissionais e gestantes, constituindo um momento de acolhida, escuta, vínculo, de compartilhamento de experiências, trocas mútuas, fortalecimento de conhecimentos e esclarecimentos de dúvidas ${ }^{(19)}$.

Estudo $^{(20)}$ desenvolvido junto a gestantes que realizavam acompanhamento pré-natal na Atenção Básica e no serviço da Clínica Escola Integrada da Faculdade Santa Maria no município de Cajazeiras-PB, que trabalhou a satisfação e as expectativas de 12 gestantes sobre a assistência pré-natal, demonstrou que a satisfação refere-se apenas aos aspectos gerais, sendo que um bom atendimento pré-natal, além de fazer acompanhamento clínico da evolução gestacional, deve ser capaz de identificar intercorrências e prestar orientações quanto à alimentação, mudanças físicas e psicológicas durante o período gestacional.

Já um estudo ${ }^{(21)}$ realizado em uma Unidade de Saúde da Família de Montes Claros-MG, que avaliou a satisfação de oito gestantes sobre a consulta de Enfermagem pré-natal, evidenciou que ao serem questionadas sobre o atendimento, destacaram o ato de examinar e o esclarecimento de dúvidas como demonstrações de bom desempenho profissional e de responsabilização de quem presta assistência. O desempenho profissional é avaliado pela gestante por meio do interesse demonstrado no exame físico, nas perguntas feitas e orientações dadas, bem como na resolutividade das condutas.

A atenção à mulher no ciclo gravídico-puerperal é indispensável para que ela possa exercer a maternidade com segurança e sem riscos. Os profissionais de saúde devem desenvolver ações interdisciplinares que favoreçam a autoconfiança e cuidado da mulher e de sua família durante a gestação e no pós-parto ${ }^{(22)}$.

Entende-se como limitação deste estudo a sua baixa possibilidade de generalização, por se tratar de uma população especifica, como a ribeirinha, mas ressalta-se que seus resultados podem contribuir para reflexão sobre as necessidades e expectativas de mulheres grávidas em contexto semelhante que, muitas vezes, têm pouco sucesso em seu pré-natal, justamente por não serem consideradas suas particularidades ambientais e culturais. 
Por meio deste estudo, pode-se afirmar que o pré-natal é considerado muito importante para as mulheres ribeirinhas e que conseguem entender a necessidade de realizá-lo. Apesar da baixa escolaridade, ficou clara sua capacidade de expressar essa importância, além de seus sentimentos e expectativas, evidenciando-se, assim, o seu empoderamento e interesse nas consultas de pré-natal.

Vale ressaltar que a maioria afirmou que sentia falta da atuação de outros profissionais e da realização de procedimentos complementares. Mencionou-se com frequência a necessidade de dentista, nutricionista, pediatra e ginecologista, além da realização dos exames no próprio serviço, evitando-se, dessa forma, idas à capital em busca desse atendimento. Nesse contexto, evidenciamse as dificuldades e os desafios ainda enfrentados por essas mulheres e ressalta-se a importância do poder público na garantia de melhorias nos serviços afastados dos meios urbanos, a exemplo da USF do Combú.

Embora essa carência em relação à integralidade da assistência no serviço de saúde esteja em seus depoimentos, ao mencionarem o atendimento da equipe na Unidade, todas se mostraram satisfeitas e, de certa forma, gratas com a equipe. Assim, percebe-se que, apesar das barreiras, a equipe atuante na Ilha do Combú soma forças para vencer as dificuldades e prestar um atendimento de qualidade, tornando-se capaz de atender às necessidades das usuárias, evidenciando-se o vínculo construído entre a equipe e as gestantes, interpretada como positiva para sua assistência, e refletida na assiduidade da maioria das grávidas nas consultas de pré-natal.

Espera-se que estes resultados ensejem mais estudos que venham suprir lacunas nessa área do conhecimento com populações específicas. Isso pode melhorar a compreensão do contexto em que as mulheres estão inseridas, suas interpretações, seus anseios e suas reais necessidades, permitindo repensar e qualificar os serviços e os cuidados de saúde ofertados às populações com essas particularidades.

\section{REFERÊNCIAS}

1. Ministério da Saúde (BR). Secretaria de Atenção à Saúde. Departamento de Atenção Básica. Atenção ao prénatal de baixo risco. [Internet]. Brasília: Ministério da Saúde; 2013[acesso em 2016 abr 06].Disponível em:http:// bvsms.saude.gov.br/bvs/publicacoes/cadernos_atencao_basica_32_prenatal.pdf.

2. Silva SN, Santos MAP, Campos NPS, Souza C, Gonzaga MFN, Pereira RSF, et al. A importância do pré-natal na prevenção da toxicemia gravídica e o papel do enfermeiro. Rev. Saúde em Foco. [Internet]2017;9(1) [acesso em 20 jul 2017]. Disponível: http://unifia.edu.br/revista_eletronica/revistas/saude_foco/artigos/ano2017/003_a_ importancia_do_pre_natal_na_prevencao_da_toxicemia.pdf.

3. Ministério da Saúde (BR). Universidade Estadual do Ceará. Humanização do parto e do nascimento. [Internet]. Brasília: Ministério da Saúde; 2014. [acesso em 2016 abr 08]. Disponível em:http://www.redehumanizasus.net/ sites/default/files/caderno_humanizasus_v4_humanizacao_parto.pdf.

4. Domingues RMSM, Hartz ZMA, Dias MAB, Leal MC. Avaliação da adequação da assistência pré-natal na rede SUS do Município do Rio de Janeiro, Brasil. Cad Saude Publica [Internet]. 2012 [acesso em 2016 abr 18]; 28(3). Disponível em:http://dx.doi.org/10.1590/S0102-311X2012000300003.

5. Fernandes ACUR, Costa DDO,CostaJM,DuarteKMM,SilvaMP,Garcia PT, et al. Saúde da mulher. [Internet]. São Luís: EDUFMA; 2017 [acesso em 2017 ago 08]. Disponível em: http://www.unasus.ufma.br/site/files/livros_isbn/ isbn_sf08.pdf.

6. Ando NM. Atuação da Medicina de Família e Comunidade em Populações Ribeirinhas. Programa de Atualização em Medicina de Família e Comunidade (PROMEF). Porto Alegre: Artmed/Panamericana; 2011.

7. Barbiani R, Junges JR, Nora CRD, Asquidamini F. A produção científica sobre acesso no âmbito do Sistema Único de Saúde do Brasil: avanços, limites e desafios. Saude Soc. [Internet]. 2014 [acesso 2017 jul 22]; 23(3). Disponível em: http://dx.doi.org/10.1590/S0104-12902014000300010. 
8. Thiry-Cherques HR. Saturação em pesquisa qualitativa: estimativa empírica de dimensionamento. Af-RevPMKT. [Internet]. 2009 [acesso em 2018 ago 18]; 4(08). Disponível em: http://www.revistapmkt.com.br/Portals/9/Edicoes/ Revista_PMKT_003_02.pdf.

9. Bardin L. Análise de conteúdo. São Paulo: Edições 70; 2011.

10. Ministério da Saúde (BR). Conselho Nacional de Saúde. Diretrizes e normas regulamentadoras de pesquisa envolvendo seres humanos. Resolução n. 466, de 12 de dezembro de 2012. Brasília; 2012.

11. Brito PE, Henrique LM. Atenção Primária à Saúde na percepção de mulheres residentes na zona rural. Rev. Esc. Enferm. USP.[Internet]. 2015 [acesso em 2018 ago 17]; 49(5). Disponível em: http://dx.doi.org/10.1590/S0080623420150000500003.

12. Landerdahl MC, Lúcia BR, Fernanda BM, Fernanda BC, Miriam OG. A percepção de mulheres sobre atenção pré-natal em uma unidade básica de saúde. Esc. Anna Nery. [Internet]. 2007 [acesso em 2016 nov 02]; 11(1). Disponível em: http://dx.doi.org/10.1590/S1414-81452007000100015.

13. Cardoso LSM, Mendes LL, Meléndez GV. Diferenças na atenção pré-natal nas áreas urbanas e rurais do Brasil: estudo transversal de base populacional. REME - Rev Min Enferm. [Internet]. 2013 [acesso em 2016 nov 10]; 17(1). Disponível em: http://www.dx.doi.org/10.5935/1415-2762.20130008.

14. Rodrigues EM, Nascimento RG, Araújo A. Protocolo na assistência pré-natal: ações, facilidades e dificuldades dos enfermeiros da Estratégia Saúde da Família. Rev. Esc. Enferm. USP. [Internet]. 2011 [acesso em 2016 jul 15]; 45(5). Disponível em: http://dx.doi.org/10.1590/S0080-62342011000500002.

15. Silva MVE. Início tardio do pré-natal: revisão integrativa da literatura [tese]. Teófilo Otoni (MG): Universidade Federal de Minas Gerais; 2012.

16. Souza ES, Nazareth IV, Gonçalves APO, Santos IMM. A look of women-mothers about prenatal care. Rev. enferm. UFPE on line. [Internet]. 2013 [acesso em 2016 jul 2]; 7(8). Disponível em:https://periodicos.ufpe.br/ revistas/revistaenfermagem/article/view/11785.

17. Conselho Regional de Enfermagem. Prefeitura, Secretaria Municipal de Saúde e Defesa Civil. Subsecretaria Geral do Rio de Janeiro. Protocolos de Enfermagem na Atenção Primária à Saúde.[Internet]. Rio de Janeiro: COREN/RJ; 2012. Disponível em: http://www.rio.rj.gov.br/dlstatic/10112/4446958/4111921/enfermagem.pdf.

18. Mendes AFP. Pré-Natal de baixo risco e puerpério: proposta de implementação de um protocolo de enfermagem [tese]. Conselheiro Lafaiete (MG): Universidade Federal de Minas Gerais; 2013. Disponível em: https://www.nescon.medicina.ufmg.br/biblioteca/imagem/4224.pdf.

19. Anversa ETR, Bastos GAN, Nunes LN, Pizzol TSD. Qualidade do processo da Assistência Pré-Natal: unidades básicas de saúde e unidade de Estratégia Saúde da Família em município no Sul do Brasil. Cad Saúde Pública. [Internet]. 2012 [acesso em 2016 nov 11];28(4). Disponível em: http://dx.doi.org/10.1590/S0102-311X2012000400018.

20. Santos AL, Radovanovic CAT, Marcon SS. Prenatal care: satisfaction and expectations. Rev. Rene. [Internet]. 2010 [acesso em 2016 out 05]; 11(n.esp). Disponível em: http://periodicos.ufc.br/rene/article/view/4662.

21. Barbosa TLA, Gomes LMX, Dias OV. O pré-natal realizado pelo enfermeiro: a satisfação das gestantes. Cogitare enferm. [Internet]. 2011 [acesso em 2016 dez 29]; 16(1). Disponível em: http://dx.doi.org/10.5380/ce.v16i1.21108.

22. Amaral FC. Assistência humanizada à gestante em unidade de saúde da família [tese]. Araçuaí (MG): Universidade Federal de Minas Gerais; 2011. Disponível em: https://www.nescon.medicina.ufmg.br/biblioteca/ imagem/3105.pdf. 